\title{
Effective equation of state of hot and dense matter in nuclear collisions around FAIR energy
}

\author{
L. Bravina ${ }^{1, a}$ and E. Zabrodin ${ }^{1,2}$ \\ ${ }^{1}$ Department of Physics, University of Oslo, PB 1048 Blindern, Oslo, Norway \\ ${ }^{2}$ Skobeltsyn Institute of Nuclear Physics, Moscow State University, RU-119991 Moscow, Russia
}

\begin{abstract}
The chemical and thermal equilibration in the central zone of heavy-ion collisions at energies around FAIR is studied within two microscopic models. Two systems are utilized for the analysis: (i) central cubic cell of fixed volume $V=125 \mathrm{fm}^{3}$ and (ii) expanding central area of uniformly distributed energy density. It is found that kinetic, thermal, and chemical equilibration of the expanding hadronic matter are nearly approached in both systems for the period of $10-18 \mathrm{fm} / c$. The expansion proceeds almost isentropically. The extracted equation of state (EOS) in $P-\varepsilon$ plane has a linear dependence $P=a \varepsilon$, where $a \equiv c_{s}^{2}$ slightly increases with the collision energy from 0.12 to 0.145 . Linear dependencies for the EOS are found also in $T-\mu_{B}$ and $T-\mu_{S}$ planes. The characteristic kinks observed in the last two phase diagrams are linked to inelastic freeze-out in the expanding fireball.
\end{abstract}

\section{Introduction}

Ultra-relativistic heavy ion collisions offer a unique opportunity to study the nuclear phase diagram at high temperatures and densities, and to search for a new state of matter, predicted by Quantum Chromodynamics (QCD), namely, a quark-gluon plasma (QGP) of deconfined quarks and gluons. Lattice QCD calculations show that as the temperature of the system crosses some critical value $T_{c}$, the energy density rapidly increases and reaches the black body limit for $T>T_{c}$, indicating that the system has turned into the QGP phase. Relaxation to local equilibrium and thermalization has become a central issue because it is implicitly or explicitly assumed in most proposed signals of the QGP. It is usually postulated that the non-equilibrium initial stage of nuclear collisions, during which shock waves, partonic jets, etc., heat the system, is considerably shorter than the characteristic hadronization times. Evidently, there must be dissipative and irreversible processes leading to equilibration. The hydrodynamic stage arises when the evolution of the system can be described in terms of local average particle number, their velocities and energies [1]. However, since the initial state of HIC's is produced far from equilibrium, its relaxation to local equilibrium should be studied within e.g. kinetic theory and/or non-equilibrium microscopic models of hadronic matter.

The microscopic models can help us to understand dynamics of relativistic heavy ion collisions and reveal, after comparison with the experimental data, the signals of the QGP formation. For our

\footnotetext{
a e-mail: larissa.bravina@fys.uio.no
} 
analysis two transport Monte Carlo models were employed: ultrarelativistic quantum molecular dynamics (UrQMD) model [2] and quark-gluon string model (QGSM) [3]. Central gold-gold collisions with zero impact parameter $b=0$ were simulated at bombarding energies $E_{\text {lab }}=11.6,20,30,40,80$ and $160 \mathrm{AGeV}$, respectively. These reactions can probe the domain of temperatures and baryon chemical potentials where, according to the lattice QCD calculations, the tricritical point of nuclear phase diagram is located [4]. Search for the tricritical point and determination of the equation of state are among the primary goals of the compressed baryon matter (CBM) experiment at GSI FAIR at energies about 20-30 AGeV [5]. But the fireball formed in the course of a heavy ion collision is not a stationary system. It expands, therefore its bulk characteristics, such as energy density and baryon density, are instantly changing. How to determine the emergence of equilibrium in this system?

The standard procedure $[6,7]$ is to compare the snapshot of particle yields and spectra in the testvolume at given time with those predicted by the statistical thermal model (SM) of hadron gas [8, 9]. The total energy, the net baryon charge, and the net strangeness extracted for a certain volume of the reaction were inserted into a system of nonlinear equations to obtain temperature, baryon chemical potential and strangeness chemical potential of an ideal hadron gas in equilibrium. If the microscopic yields and transverse momentum spectra of particles are close to that provided by the statistical model, the matter in the cell is considered to be at least in the vicinity of equilibrium. Then its equation of state and other thermodynamic characteristics can be derived and studied.

This analysis was further modified in [10]. In addition to a fixed-size cell the concept of expanding volume of uniformly distributed energy density was implemented. Here the whole volume of the fireball is subdivided into rather small cells. Suppose that at certain time the energy densities of $n$ inner cells are similar. The verification of the equilibrium conditions is repeated after a timestep of $\Delta t=1 \mathrm{fm} / c$. If the energy densities of $m$ outer cells become the same as those of the $n$ inner cells, the new volume to be checked for the fulfillment of local equilibrium conditions consists now of $n+m$ cells, and so on.

The paper is organized as follows. After a brief introduction of basic principles of microscopic transport models UrQMD and QGSM in Sec.2, the statistical model of an ideal hadron gas is formulated in Sec.3. Section 4 presents study of relaxation of fireballs produced in central heavy ion collisions at different bombarding energies to the equilibrium. Effective EOS in the form $P=a \varepsilon$ is extracted in Sec.5. Analysis is extended to $T-\mu_{B}$ and $T-\mu_{S}$ diagrams as well. Conclusions are drawn in Sec.6.

\section{Microscopic models}

Both UrQMD and QGSM are formulated as Monte-Carlo event generators allowing to perform a careful analysis of the measurable quantities by introducing all necessary experimental cuts. The models are designed to describe hadronic, hadron-nucleus, and nuclear collisions in a broad energy range. In the hadronic sector both models treat the production of new particles via formation and fragmentation of specific colored objects, strings. Strings are uniformly stretched, with constant string tension $\kappa \approx 1 \mathrm{GeV} / \mathrm{fm}$, between the quarks, diquarks and their antistates. The excited string is fragmenting into pieces via the Schwinger-like mechanism of $q \bar{q}$-pair production, and the produced hadrons are uniformly distributed in the rapidity space.

In the UrQMD model the longitudinal excitation of strings is employed, and the string masses arise from momentum transfer. In the QGSM model the string masses appear due to the color exchange mechanism, and strings are stretched between the constituents belonging to different hadrons. Also, the fragmentation functions which determine the energy, momentum, and type of the hadrons produced during the string decay, are different in the models. Tables of the experimentally available 
information, such as hadron cross sections, resonance widths and decay modes, are implemented in the models. If this information is lacking, the one-boson exchange model, detailed balance considerations and isospin symmetry conditions are employed. The propagation of particles is governed by Hamilton equation of motion, and both models use the concept of hadronic cascade for the description of $h A$ and $A+A$ interactions. Due to the uncertainty principle newly produced particles can interact further only after a certain formation time. Only hadrons containing the valence quarks can interact immediately with the reduced cross section $\sigma=\sigma_{q N}$. The Pauli principle is taken into account via the blocking of the final state, if the outgoing phase space is occupied.

\section{Statistical model}

If the system is in thermal and chemical equilibrium, its macroscopic characteristics are fully determined by particle distribution functions

$$
f\left(p, m_{i}\right)=\left[\exp \left(\frac{\sqrt{p^{2}+m_{i}^{2}}-\mu_{\mathrm{B}} B_{i}-\mu_{\mathrm{S}} S_{i}}{T}\right) \pm 1\right]^{-1},
$$

where $p, m_{i}$ and $d_{i}$ is the momentum and mass of the hadron species $i$, respectively. Sign + stands for fermions and - for bosons. One has to know just three parameters, namely, temperature $T$ and chemical potentials assigned to the conserved charges: baryon chemical potential $\mu_{\mathrm{B}}$ and strangeness chemical potential $\mu_{\mathrm{S}}$. Chemical potential of $i$-th hadron depends on baryon and strangeness content $\mu_{i}=B_{i} \mu_{\mathrm{B}}+S_{i} \mu_{\mathrm{S}}$. Then the expressions for particle number density $n_{i}$, energy density $\varepsilon_{i}$ and pressure $P$ read

$$
\begin{aligned}
n_{i} & =\frac{g_{i}}{2 \pi^{2}} \int_{0}^{\infty} p^{2} f\left(p, m_{i}\right) d p \\
\varepsilon_{i} & =\frac{g_{i}}{2 \pi^{2}} \int_{0}^{\infty} p^{2} \sqrt{p^{2}+m_{i}^{2}} f\left(p, m_{i}\right) d p \\
P & =\frac{g_{i}}{2 \pi^{2}} \int_{0}^{\infty} p^{2} d p \frac{p^{2}}{3\left(p^{2}+m_{i}^{2}\right)^{1 / 2}} f\left(p, m_{i}\right) .
\end{aligned}
$$

with $g_{i}$ being the spin-isospin degeneracy factor. The entropy density $s_{i}$ can be calculated either via the distribution function as

$$
s_{i}=-\frac{g_{i}}{2 \pi^{2}} \int f\left(p, m_{i}\right)\left[\ln f\left(p, m_{i}\right)-1\right] p^{2} d p,
$$

or from the Gibbs thermodynamic identity

$$
T s_{i}=\varepsilon_{i}+P_{i}-\mu_{B} \rho_{B_{i}}-\mu_{S} \rho_{S_{i}} .
$$

The total energy density $\varepsilon$, baryon density $\rho_{B}$ and strangeness density $\rho_{S}$ calculated microscopically within the cell at time $t$ are inserted into the set of nonlinear equations

$$
\begin{aligned}
\varepsilon & =\frac{1}{V} \sum_{i} E_{i}^{\mathrm{SM}}\left(T, \mu_{\mathrm{B}}, \mu_{\mathrm{S}}\right), \\
\rho_{\mathrm{B}} & =\frac{1}{V} \sum_{i} B_{i} \cdot N_{i}^{\mathrm{SM}}\left(T, \mu_{\mathrm{B}}, \mu_{\mathrm{S}}\right), \\
\rho_{\mathrm{S}} & =\frac{1}{V} \sum_{i} S_{i} \cdot N_{i}^{\mathrm{SM}}\left(T, \mu_{\mathrm{B}}, \mu_{\mathrm{S}}\right) .
\end{aligned}
$$


to determine temperature $T$, baryon chemical potential $\mu_{B}$ and strangeness chemical potential $\mu_{S}$. After that all characteristics of the system in equilibrium are known and particle spectra can be compared with those obtained from microscopic model calculations.

\section{Relaxation to equilibrium}

The most appropriate area to look for the equilibrium is the central cell in the center-of-mass (CM) frame. In this case the net collective flow of particles is essentially zero. The cell should be neither too large because of possible density impurities nor too small to validate the statistical description. Previous studies revealed [6,7] that the cubic cell with volume $V=125 \mathrm{fm}^{3}$ is well suited for our analysis. To study the early stage of the evolution and the onset of the equilibrium, the central cell was further subdivided into the smaller ones, embedded one into another. The energy density was calculated for each cell. In case the energy densities of the inner and outer cells are similar, the latter becomes a new test-volume. Thus one follows the expansion of the area of homogeneously distributed energy.

The equilibrium in the cell cannot set in earlier than the time needed for Lorentz contracted nuclei to pass through each other and leave the cell. After that the pressure isotropy should be checked. Figure 1 shows the time evolution of pressure in transverse $(x)$ and longitudinal $(z)$ directions calculated for $\mathrm{A}+\mathrm{A}$ collisions at energies from $11.6 \mathrm{AGeV}$ to $158 \mathrm{AGeV}$.

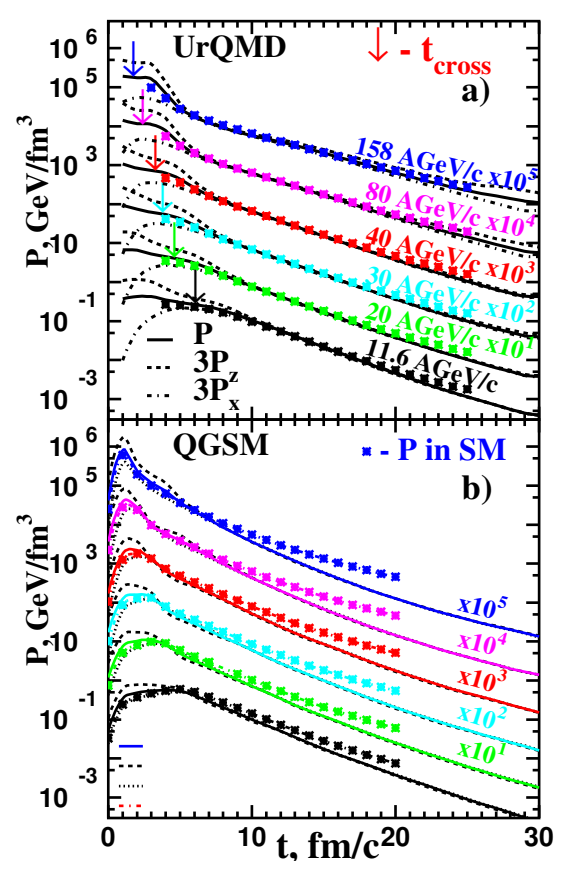

Figure 1. Time evolution of pressure components in Figure 2. Energy spectra of hadrons at $t=13 \mathrm{fm} / c$ the central cell of $\mathrm{Au}+\mathrm{Au}$ collisions at energies from and $t=10 \mathrm{fm} / c$ in (a) UrQMD and (b) QGSM calcula11.6 AGeV to $158 \mathrm{AGeV}$ in (a) UrQMD and (b) QGSM tions. Lines correspond to the fit to Boltzmann distribucalculations.

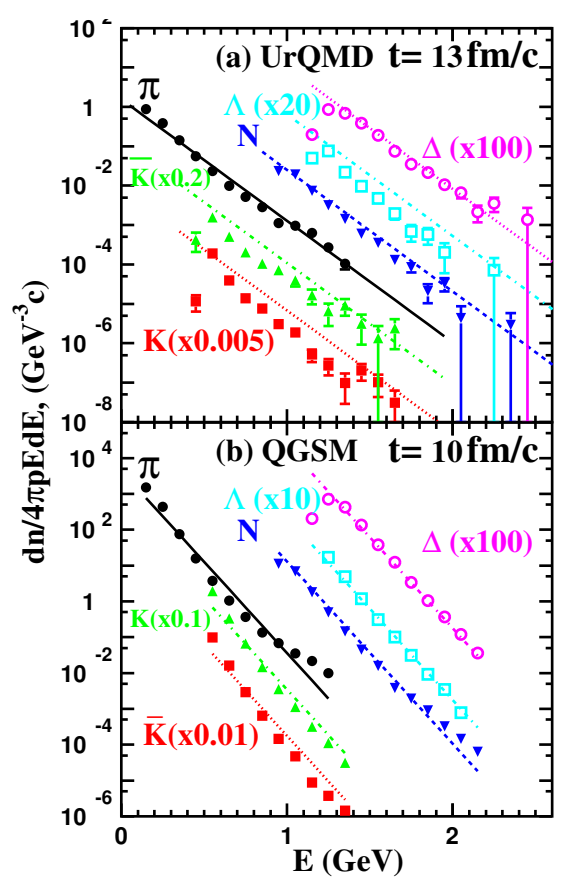

tion with single temperature $T$. 
These components become very close (within the 5\%-limit of accuracy) to each other after the certain time. The higher the bombarding energy, the faster the convergence, and vice versa. Comparison with the pressure calculated in the SM (also plotted in Fig. 1) shows a good agreement between microscopic and macroscopic calculations. The preequilibrium stage in the cell holds for a period of about $10-12 \mathrm{fm} / \mathrm{c}$ (Fig. 1). Then the system develops again the anisotropy in the pressure and velocity sectors due to significant reduction of number of collisions between particles.
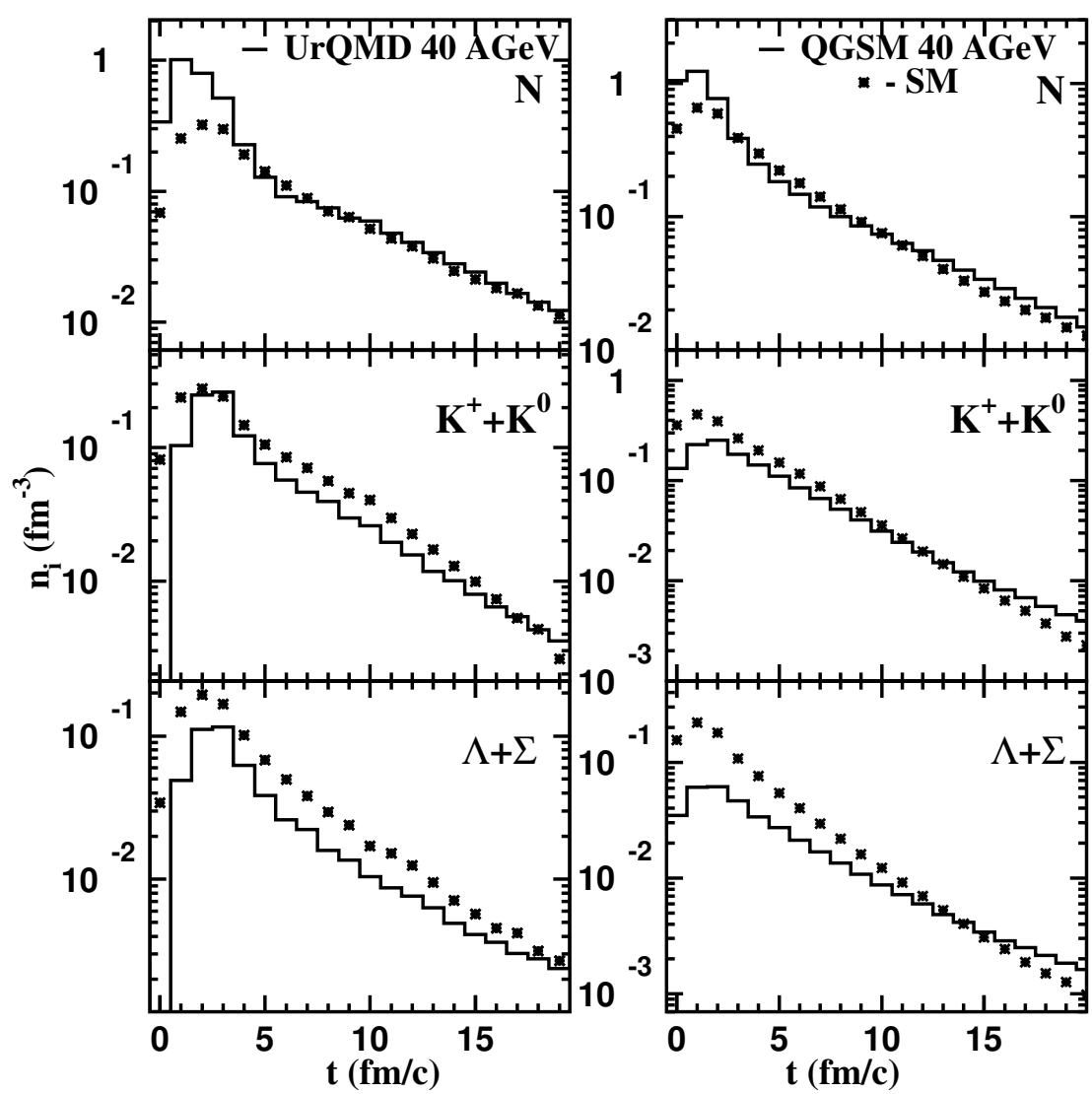

Figure 3. Hadron yields in the central cell with $V=125 \mathrm{fm}^{3}$ of central Au+Au collisions at $40 \mathrm{AGeV}$ in UrQMD (left) and QGSM (right) calculations.

Isotropy of the pressure gradients is just the necessary prerequisite of local equilibrium. Two other conditions demand fulfillment of thermal and chemical equilibrium. The energy spectra $d N / 4 \pi p E d E$ shown in Fig. 2 are fitted to Boltzmann distribution. In both models the shapes of the spectra are close to the exponential ones, corresponding to unique temperature $T$. This is taken as evidence of thermal equilibration in the system. The yields of hadron species in the central cell of Au+Au collisions at $E_{l a b}=40 \mathrm{AGeV}$ at the moment $t=13 \mathrm{fm} / c$ are displayed in Fig. 3. Statistical model results are plotted onto the distributions also. One can see that for a certain period the calculations of microscopic and macroscopic models are close to each other, i.e. chemical equilibrium is attained. 


\section{Equation of State}

How dense is the compressed baryonic matter? Here it is important to mention two factors. First, the density estimations are very sensitive to the tested volume, especially at the early stage. Figures 4 and 5 indicate that for the central cell of relatively big volume $V=125 \mathrm{fm}^{3}$ the baryon density in both models cannot exceed $5 \rho_{0}$ at any energy in question. In contrast, for the small cell of volume $V=0.125 \mathrm{fm}^{3}$ the ratio $\rho_{B} / \rho_{0}$ can be as high as 15 at $E_{\text {lab }}=40 \mathrm{AGeV}$, as shown in bottom plots of these figures. Such high densities were reported in [11], where calculations of several microscopic and macroscopic models were compared. In our opinion this is a pure kinematic effect. When two Lorentz-contracted nuclei pass through each other, the apparent baryonic density can be quite high. By no means the whole system is far from equilibrium. Solid lines represent the calculations related to equilibrated matter at $t \approx 10 \mathrm{fm} / c$. We see that the baryon density in the cell at this time is about twice of normal nuclear density.
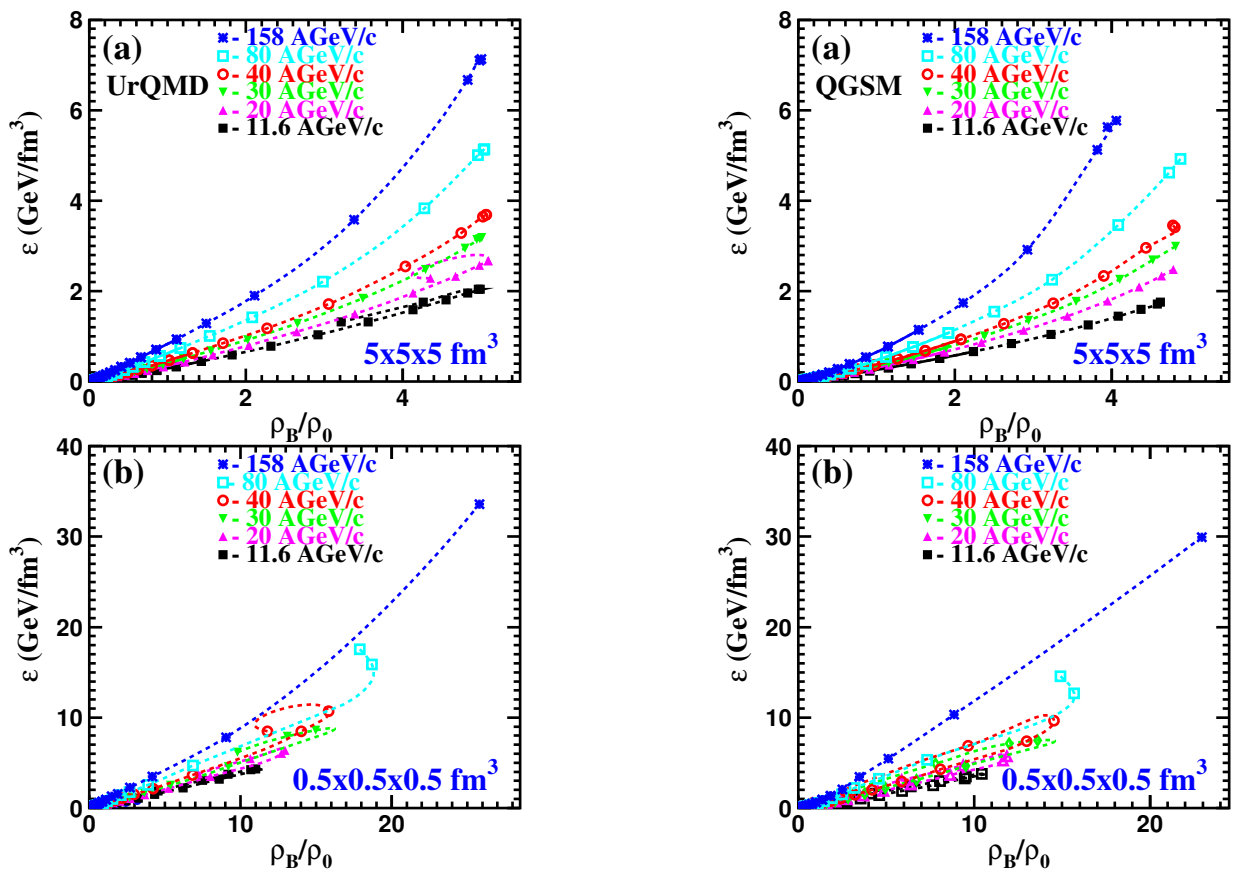

Figure 4. Time evolution of total energy density vs. Figure 5. Time evolution of total energy density vs. baryon density (in units of $\rho_{0}$ ) in the central UrQMD baryon density (in units of $\rho_{0}$ ) in the central QGSM cell cell of volume (a) $125 \mathrm{fm}^{3}$ and (b) $0.125 \mathrm{fm}^{3}$ for differ- of volume (a) $125 \mathrm{fm}^{3}$ and (b) $0.125 \mathrm{fm}^{3}$ for different ent bombarding energies. bombarding energies.

Isentropic expansion of relativistic fluid is one of the main postulates of Landau hydrodynamic theory [1] of multiparticle production. Though conditions in the cell are instantly changing, it is possible to check the behavior of the entropy per baryon. Within the 5\% accuracy limit, this ratio is nearly conserved in the equilibrium phase of expansion, see Fig. 6. The entropy densities obtained for the cell in both models are very close to each other, but, because of the difference in net-baryon sector, the ratio $s / \rho_{\mathrm{B}}$ in UrQMD is about $15-20 \%$ larger than that in QGSM. Recall that both the pressure isotropy and the conservation of $s / \rho_{\mathrm{B}}$ supports the application of hydrodynamics. 

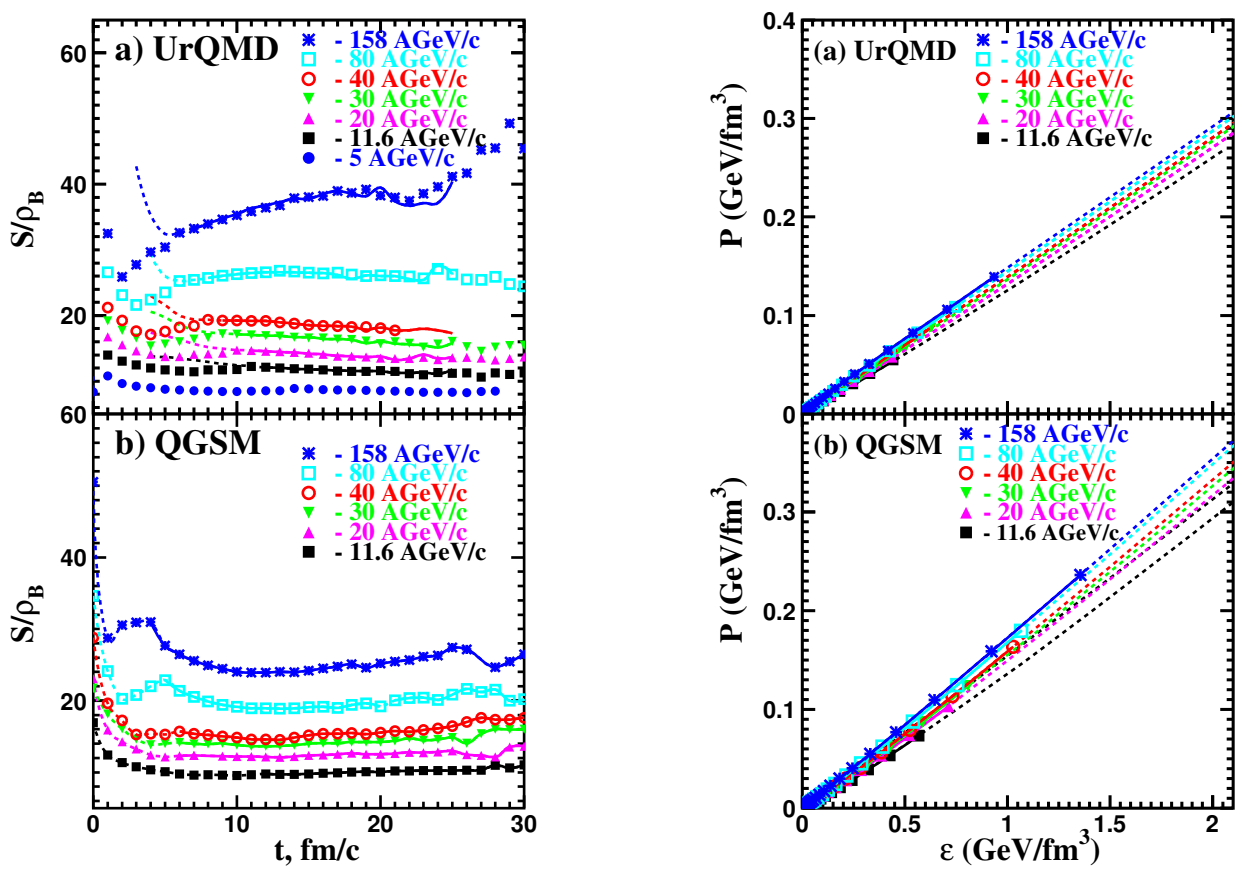

Figure 6. Time evolution of entropy per baryon $S / \rho_{\mathrm{B}}$ in Figure 7. Time evolution of the pressure $P$ and the enin (a) UrQMD and (b) QGSM calculations of Au+Au ergy density $\varepsilon$ in the central $125 \mathrm{fm}^{3}$ cell in (a) UrQMD collisions at energies from 11.6 AGeV to $158 \mathrm{AGeV}$. and (b) QGSM calculations.

Dashed lines correspond to the non-equilibrium stage of the reaction, solid lines represent the equilibrium phase.

Without the equation of state, which links pressure of the system to its energy density, the system of hydrodynamic equations is incomplete. The corresponding plot is presented in Fig. 7. For all energies in question and for both models the dependence of pressure on energy density is remarkably linear. Thus the EOS has a rather simple form $P(\varepsilon)=a \varepsilon$, where $a$ varies from 0.12 at $E_{l a b}=11.6 \mathrm{AGeV}$ to 0.145 at $E_{l a b}=158 \mathrm{AGeV}$. The slope parameter is related to the speed of sound in the system as $a \equiv c_{s}^{2}$. Both models indicate that the increase of the slope parameter with rising bombarding energy is changing at $E_{l a b}=40 \mathrm{AGeV}$. After this energy threshold the sonic velocity is less sensitive to collision energy [10].

The obtained results are in line with other estimates. For instance, the presence of resonances in hadron spectrum should reduce the speed of sound. In this case the EOS can be coined in the form [12]

$$
\varepsilon=(\alpha+4) P,
$$

where $2 \leq \alpha \leq 3$. It means that $\frac{1}{7} \leq c_{s}^{2} \leq \frac{1}{6}$. At RHIC energies $\sqrt{s}=200 \mathrm{GeV}$ PHENIX collaboration extracted the value $c_{s} \approx 0.35 \pm 0.05$ [13] from the analysis of elliptic flow. This value $c_{s}^{2}=0.12 \pm 0.03$ is also very close to our result. All obtained results favor the quite soft EOS.

The time evolution of the central cell in $T-\mu_{B}$ plane is shown in Fig. 8. The increase of temperature with the initial collision energy is accompanied by the drop of baryo-chemical potential in line with the statistical model analysis of the freeze-out conditions at various energies [9]. At energies above 

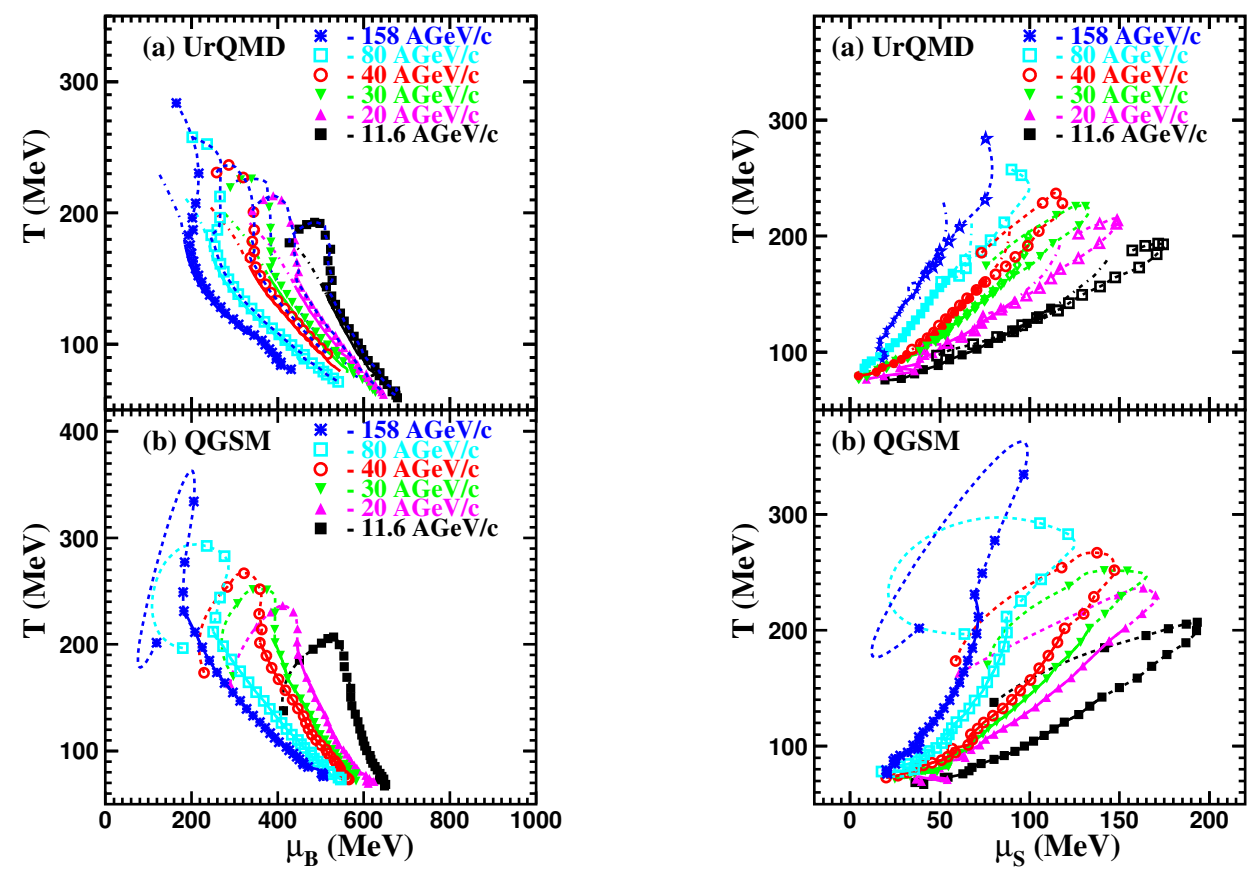

Figure 8. Time evolution of the temperature $T$ and Figure 9. The same as Fig. 8 but for the temperature baryo-chemical potential $\mu_{B}$ pressure $P$ in (a) UrQMD - strangeness chemical potential plane. See text for deand (b) QGSM calculations. Symbols and dashed tails.

curves present the evolution in a cell with increasing volume, whereas dash-dotted and full curves are related to the fixed-volume cell.

$40 \mathrm{AGeV}$ the temperature in the cell at the onset of equilibrium is close to the area of phase transition predicted by the MIT bag model for ideal quark-gluon plasma phase with $m_{S}=0$ [6]. For the "expanding cell" we see distinctly the kink (or "knee"), corresponding to the beginning of the stage of kinetic equilibrium for all energies in question. The entropy per baryon ratio becomes constant here already at $t=4 \mathrm{fm} / c$. It is worth mentioning that similar kinks were observed in several quite different models, such as (i) lattice QCD simulations for 2-flavor medium [14]; (ii) $\sigma-\omega-\rho$ model with scaled hadron masses [15]; (iii) chiral fluid dynamics model with Polyakov loop [16] also along the lines of constant entropy per baryon. Despite the similarities, the origin of the phenomenon in microscopic calculations might not necessarily be the same. For instance, it can be connected to transition from strings as dominant mechanism of particle production to (quasi)elastic collisions, i.e. to inelastic freeze-out.

Evolution of temperature with strangeness chemical potential is shown in Fig.9. Again, the kinks emerge exactly at the transition from non-equilibrium phase to the equilibrium one. In contrast to baryo-chemical potential, which increases with time, the strangeness chemical potential tends to decrease. The evolution of both potentials with temperature proceeds almost linearly.

Final remark. It looks a bit disappointing that equilibration in the fireball sets in quite late at $t \approx 10 \mathrm{fm} / c$. However, many distributions presented here (see, e.g. EOS-plot or entropy-per-baryon ratio) indicate that quasi-equilibrium description can be applicable much earlier, at $t \approx 3-4 \mathrm{fm} / \mathrm{c}$. In 
this sense our work supports the results of [17], where it was shown that the hydrodynamic description under a certain conditions can be applicable to systems which are not in local equilibrium. This interesting and important question should be elaborated in future.

\section{Conclusions}

The following conclusions can be drawn from our study. Both UrQMD and QGSM predict the formation of chemically and thermally equilibrated hot and dense nuclear matter in central heavy ion collisions at energies from $11 \mathrm{AGeV}$ to $158 \mathrm{AGeV}$. The equilibrium phase lasts about $10-15 \mathrm{fm} / c$. During this period the expansion of matter in the central cell proceeds isentropically with constant entropy per baryon, thus supporting the application of hydrodynamics. The EOS has a linear form $P=c_{s}^{2} \varepsilon$, where the speed of sound rises to $c_{s}^{2}=0.14$ at $E_{l a b}=40 \mathrm{AGeV}$ and then slowly increases to 0.145 at SPS and RHIC energies. The knee-structure which appears distinctly in $T-\mu_{B}$ and $T-\mu_{S}$ phase diagrams in calculations for expanding zone of uniformly distributed energy is likely to be related with the chemical freeze-out in microscopic models. However, other possibilities, e.g. phase transition, etc. are not ruled out.

\section{References}

[1] L.D. Landau, Izv. Akad. Nauk SSSR, Ser. Fiz. 17, 51 (1953) 51 (in Russian);

S.Z. Belenkij, L.D. Landau, Nuovo Cimento Suppl. 3, 15 (1956)

[2] S.A. Bass et al., Prog. Part. Nucl. Phys. 41, 255 (1998);

M. Bleicher et al., J. Phys. G25, 1859 (1999)

[3] N.S. Amelin, L.V. Bravina, Sov. J. Nucl. Phys. 51 (1990) 133;

N.S. Amelin, L.V. Bravina et al., Phys. Rev. C47, 2299 (1993)

[4] S. Ejiri et al., Prog. Theor. Phys. Suppl. 153, 118 (2004);

Z. Fodor, S.D. Katz, JHEP 0404, 050 (2004)

[5] W. Henning, Nucl. Phys. A734, 654 (2004)

[6] L.V. Bravina et al., Phys. Rev. C60, 024904 (1999);

L.V. Bravina et al., Phys. Lett. B434, 379 (1998);

L.V. Bravina et al., J. Phys. G25, 351 (1999)

[7] L.V. Bravina et al., Phys. Rev. C63, 064902 (2001);

L.V. Bravina et al., Nucl. Phys. A698, 383c (2002);

L.V. Bravina et al., J. Phys. G27, 421 (2001)

[8] M. Gazdzicki, M. Gorenstein, Acta Phys. Polon. B30, 2705 (1999)

[9] J. Cleymans, K. Redlich, Phys. Rev. C60, 054908 (1999)

[10] L.V. Bravina et al., Phys. Rev. C78, 014907 (2008);

L.V. Bravina et al., J. Phys. G36, 064065 (2009)

[11] I.C. Arsene et al., Phys. Rev. C75, 034902 (2007)

[12] E. Shuryak, Sov. J. Nucl. Phys. 16, 220 (1973)

[13] A. Adare et al. (PHENIX Collaboration), Phys. Rev. Lett. 98, 162301 (2007).

[14] S. Ejiri, F. Karsch, E. Laermann, C. Schmidt, Phys. Rev. D73, 054506 (2006)

[15] A. Khvorostukhin et al., Nucl. Phys. A791, 180 (2007)

[16] C. Herold et al., Nucl. Phys. A925, 14 (2014)

[17] Ph. Mota, T. Kodama, R.D. de Souza, J. Takahashi, Eur. Phys. J. A48, 165 (2012) 